resistance is still generally considered novel. We have reported on the use of MALDI-TOF MS to identify vancomycin resistant enterococci (VRE) and the implementation of this method in the routine clinical microbiology laboratory. In this presentation this work will be reviewed in addition to recent advances in use of MALDI-TOF MS to identify other forms of antimicrobial resistance and hence contribute to infection control.

\section{RAPID DETECTION OF}

\section{CARBAPENEMASE-PRODUCING}

\section{ENTEROBACTERIACEAE USING MALDI-TOF MS}

James Knox ${ }^{1}$, Snehal Jadhav ${ }^{2}$, Danielle Sevior ${ }^{3}$, Alex Agyekum $^{4}$, Margaret Whipp ${ }^{5}$, Lynette Waring ${ }^{1}$, Jonathan Iredell $^{4,6}$, Enzo Palombo ${ }^{2}$

${ }^{1}$ Microbiology Department, Melbourne Pathology, ${ }^{2}$ Department of Chemistry and Biotechnology, Swinburne University of Technology, Melbourne, Vic, ${ }^{3}$ BioMérieux Australia Pty Ltd, Sydney, ${ }^{4}$ Westmead Millennium and Marie Bashir Institutes, University of Sydney, NSW, ${ }^{5}$ Microbiological Diagnostic Unit, Public Health Laboratory, University of Melbourne, Vic, and ${ }^{6}$ Centre for Infectious Diseases and Microbiology, Westmead Hospital, Sydney, NSW, Australia

Background: The global emergence of carbapenemase-producing Enterobacteriaceae (CPE) is a major threat to public health. The development of rapid and more accurate methods for their routine laboratory detection is a vital component of control efforts.

Method: A simple technique for the detection of CPE by matrix-assisted laser desorption ionisation-time of flight mass spectrometry (MALDI-TOF MS) was developed for use in the clinical laboratory. A collection of 105 genetically-characterised Enterobacteriaceae comprising a variety of carbapenemase-producing strains and non-carbapenemase-producing strains were tested using this method: an imipenem hydrolysis assay followed by mass spectrometry to detect the breakdown of the antibiotic. For comparison, each strain was also subjected to the Carba NP test. Sensitivity and specificity of the two methods for detection of carbapenemase production were compared.

Results: Using PCR as the reference method, both tests demonstrated a sensitivity of $87 \%$ and a specificity of $100 \%$.

Conclusions: MALDI-TOF MS offers a potential alternative for the rapid detection of $\mathrm{CPE}$ in the clinical laboratory. The imipenem hydrolysis assay described could be further optimised for this purpose.

Acknowledgement: This work was originally published in Knox J, Jadhav S, Sevior D, et al. Phenotypic detection of carbapenemaseproducing Enterobacteriaceae by use of matrix-assisted laser desorption ionization-time of flight mass spectrometry and the Carba NP Test. J Clin Microbiol 2014; 52: 4075-7. 